CRYSTALLOGRAPHIC COMMUNICATIONS

ISSN 2056-9890

Received 11 December 2018

Accepted 16 April 2019

Edited by G. S. Nichol, University of Edinburgh, Scotland

Keywords: crystal structure; siloxide.

CCDC reference: 1910627

Supporting information: this article has supporting information at journals.iucr.org/e

\section{Potassium chloridotris(hypersiloxy)aluminate dimer}

\author{
Andrew P. Purdy ${ }^{\mathrm{a} *}$ and Raymond J. Butcher ${ }^{\mathrm{b}}$
}

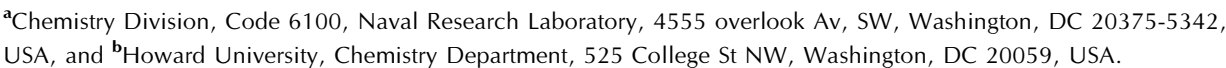
*Correspondence e-mail: andrew.purdy@nrl.navy.mil

The tris(trimethylsilylsiloxide) ligand, also known as hypersiloxide, is an extremely bulky group. In an attempt to make the monomeric $\mathrm{Al}\left(\mathrm{OSi}\left(\mathrm{SiMe}_{3}\right)_{3}\right)_{3}, \mathrm{AlCl}_{3}$ was combined with 3 equiv. of potassium hypersiloxide. The crystalline product isolated $\left(40 \%\right.$ yield), namely di- $\mu_{3}$-chlorido-bis $\left[\mu_{2^{-}}\right.$ tris(trimethylsilyl)silanolato]tetrakis[tris(trimethylsilyl)silanolato]dialuminiumdipotassium, $\left[\mathrm{Al}_{2} \mathrm{~K}_{2} \mathrm{Cl}_{2}\left(\mathrm{C}_{19} \mathrm{H}_{27} \mathrm{OSi}_{4}\right)_{6}\right]$, is a $\mathrm{KCl}$ adduct of aluminium tris(hypersilyloxide) that is dimerized through a planar $\mathrm{K}_{2} \mathrm{Cl}_{2}$ ring, with $\mathrm{K}-\mathrm{Cl}$ distances of $3.1131(8)$ and $3.319(3) \AA$, and ring angles of $77.41(2)$ and $102.60(2)^{\circ}$. This ring is on an inversion center, and there is no supramolecular coordination.

\section{Chemical context}

Alkoxides and siloxides of electropositive metals with empty $p$ and $d$ orbitals, such as aluminum, tend to be dimers, trimers, or higher oligomers, from coordination between the alkoxy oxygens and the metal atom on another molecule. One way to prepare monomeric homoleptic compounds of such metals is by using extremely bulky ligands that prevent intermolecular coordination. One of the bulkiest siloxide ligands known is tris(trimethylsilyl)silyl, also known as hypersilyl (Niemeyer, 2006). While the hypersiloxide (Boyle et al., 2018) could potentially have enough steric bulk to enable a homoleptic monomeric aluminum alkoxide to be prepared, we isolated a dimer of a $\mathrm{KCl}$ addition compound, dimerized through potassium and chlorine. The title compound was the only product that crystallized, but there were other products present, and these products decomposed during an attempt at sublimation. If less bulky, and thus more volatile, aluminum siloxides or alkoxides can form a stable soluble $\mathrm{KCl}$ adducts, which could lead to a means of solubilizing alkali metal halides from organometallic reactions without using protic solvents.

\section{Structural commentary}

Each tris(hypersiloxy)chloroaluminate ion is joined into the dimeric structure by $\mathrm{a} \mathrm{K}^{+}$ion coordinated to the chlorine atoms and one of the siloxy oxygen atoms, O1 (Fig. 1). The $\mathrm{K}_{2} \mathrm{Cl}_{2}$ ring is constrained by symmetry to be planar as it is on an inversion center, but the adjoined four-membered K1-Cl1O1-Al1 rings deviate slightly from planarity, with the angle between the $\mathrm{K}_{2} \mathrm{Cl}_{2}$ plane and mean $\mathrm{K} 1-\mathrm{Cl} 1-\mathrm{Al1}-\mathrm{O} 1$ planes being $47.8(1)^{\circ}$. The coordination around the aluminum atom is approximately tetrahedral with angles ranging from 100.32 (6) to $114.63(8)^{\circ}$. Both $\mathrm{Al}-\mathrm{O}$ bonds to the terminal 
siloxy ligands are $1.711(1) \AA$, and the Al1-O1 bond is slightly longer at 1.746 (1) $\AA$, within the normal range for aluminum siloxides. In a series of aluminum complexes of silanediols, terminal $\mathrm{Al}-\mathrm{O}$ bonds ranged from 1.709 (2) to 1.781 (4) $\AA$, and all $\mathrm{Al}-\mathrm{O}$ bonds to siloxide oxygen atoms bridging between aluminum atoms were longer than $1.8 \AA$ (Krempner et al., 2007). Likewise, the aluminum phenylsiloxide $\mathrm{Al}\left(\mathrm{OSiPh}_{3}\right)_{3}(\mathrm{THF})$ with all terminal siloxides has $\mathrm{Al}-\mathrm{O}$ bond lengths ranging from $1.696(5)$ to $1.709(5) \AA$ (Apblett et al. 1992).

$\mathrm{K}_{2} \mathrm{Cl}_{2}$ rings in organometallic complexes can be isolated or part of larger $\mathrm{K}-\mathrm{Cl}$ assemblages. For isolated $\mathrm{K}_{2} \mathrm{Cl}_{2}$ rings, both planar and puckered rings are known, with planar rings lying on an inversion center the most common. The $\mathrm{K}-\mathrm{Cl}$ distances in the title compound at 3.1131 (8) and 3.319 (3) $\AA$ are normal for this kind of feature, and the ring angles of 77.41 (2) and $102.60(2)^{\circ}$ around $\mathrm{K}$ and $\mathrm{Cl}$, respectively, are typical for this kind of ring. Reported examples of similar features are in $\mathrm{K}[\mathrm{GaCl}]\left\{\mathrm{Co}_{2}(\mathrm{CO})_{6}(\mu-\mathrm{CO})\right\}\left\{\mathrm{Co}(\mathrm{CO})_{4}\right\}$, which has $\mathrm{K}-\mathrm{Cl}$ distances of 3.129 (1) and 3.197 (1) $\AA$ and angles of 73.82 (3) and 106.18 (3) (Leiner et al., 2002), and in a chloroaluminate complex $[\mathrm{K}-\mathrm{Cl}$ distances of 3.160 (2) and 3.192 (1) $\AA$ and angles of 75.66 (3) and 104.24 (4) ${ }^{\circ}$; Abdalla, et al., 2015].

\section{Supramolecular features}

The molecule is completely surrounded by ligands and thus there are no supramolecular features.

\section{Database survey}

A search of the Cambridge Structural Database (V 5.38, update May 2017; Groom et al., 2016) for Al siloxides produced 255 hits, and the most common moieties have either phenyl or methyl attached to the silicon. However, no

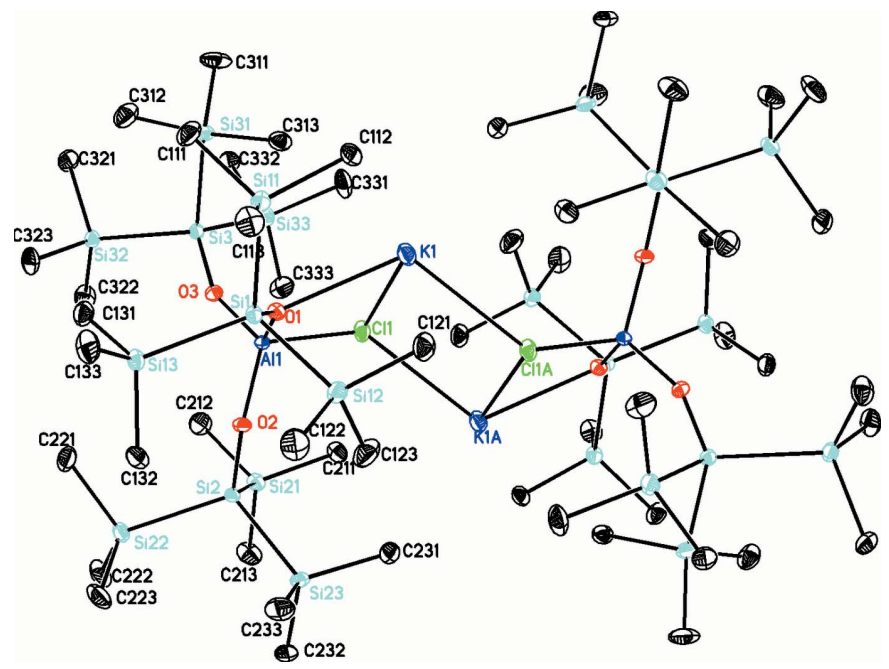

Figure 1

Diagram of the title compound, with displacement ellipsoids drawn at the $30 \%$ probability level. $\mathrm{H}$ atoms omitted for clarity. Symmetry code A: $1-x, 1-y, 1-z$.
Table 1

Experimental details.

\begin{tabular}{|c|c|}
\hline \multicolumn{2}{|l|}{ Crystal data } \\
\hline Chemical formula & {$\left[\mathrm{Al}_{2} \mathrm{~K}_{2} \mathrm{Cl}_{2}\left(\mathrm{C}_{19} \mathrm{H}_{27} \mathrm{OSi}_{4}\right)_{6}\right]$} \\
\hline$M_{\mathrm{r}}$ & 1785.05 \\
\hline Crystal system, space group & Monoclinic, $P 2_{1} / n$ \\
\hline Temperature (K) & 173 \\
\hline$a, b, c(\AA)$ & $\begin{array}{l}18.8247(3), 13.8850(2), \\
22.2056(4)\end{array}$ \\
\hline$\beta\left(^{\circ}\right)$ & $109.413(2)$ \\
\hline$V\left(\AA^{3}\right)$ & $5474.14(17)$ \\
\hline$Z$ & 2 \\
\hline Radiation type & $\mathrm{Cu} K \alpha$ \\
\hline$\mu\left(\mathrm{mm}^{-1}\right)$ & 4.16 \\
\hline Crystal size $(\mathrm{mm})$ & $0.37 \times 0.31 \times 0.24$ \\
\hline \multicolumn{2}{|l|}{ Data collection } \\
\hline Diffractometer & Agilent Xcalibur Eos Gemini \\
\hline Absorption correction & $\begin{array}{l}\text { Multi-scan (CrysAlis PRO; } \\
\text { Agilent, 2014) }\end{array}$ \\
\hline$T_{\min }, T_{\max }$ & $0.441,1.000$ \\
\hline $\begin{array}{l}\text { No. of measured, independent and } \\
\text { observed }[I>2 \sigma(I)] \text { reflections }\end{array}$ & $25914,10450,8699$ \\
\hline$R_{\text {int }}$ & 0.035 \\
\hline$(\sin \theta / \lambda)_{\max }\left(\AA^{-1}\right)$ & 0.615 \\
\hline \multicolumn{2}{|l|}{ Refinement } \\
\hline$R\left[F^{2}>2 \sigma\left(F^{2}\right)\right], w R\left(F^{2}\right), S$ & $0.042,0.117,1.04$ \\
\hline No. of reflections & 10450 \\
\hline No. of parameters & 433 \\
\hline $\mathrm{H}$-atom treatment & $\mathrm{H}$-atom parameters constrained \\
\hline$\Delta \rho_{\max }, \Delta \rho_{\min }\left(\mathrm{e} \AA^{-3}\right)$ & $0.76,-0.28$ \\
\hline
\end{tabular}

Computer programs: CrysAlis PRO (Agilent, 2014), SHELXT (Sheldrick, 2015a), SHELXL2016 (Sheldrick, 2015b) and SHELXTL (Sheldrick, 2008).

aluminum complexes of the tris(trimethylsilyl)siloxy ligand were found. The closest analogs are some complexes of chelating silanediols $\mathrm{HO}\left(\mathrm{Me}_{3} \mathrm{Si}\right)_{2} \mathrm{SiSi}\left(\mathrm{SiMe}_{3}\right)_{2} \mathrm{OH}$ and $\mathrm{HO}(\mathrm{Me})\left[\left(\mathrm{Me}_{3} \mathrm{Si}\right)_{3} \mathrm{Si}\right] \mathrm{SiSi}\left[\mathrm{Si}\left(\mathrm{SiMe}_{3}\right)_{3}\right](\mathrm{Me}) \mathrm{OH}$ (Krempner et al., 2007). A search for a tri(organosilyl)siloxy ligand attached to a metal produced six unique hits. These include $\mathrm{Fe}^{\mathrm{II}}$ and Co $^{\text {II }}$ complexes (Kornev et al., 1997 and Chesnokova et al., 2002), lanthanide(III) complexes (Kornev et al., 1999), and $\mathrm{Ta}^{\mathrm{V}}$ complexes (Wu et al., 2002).

\section{Synthesis and crystallization}

Hypersilanol was prepared by literature methods (Gilman \& Harrell, 1966). The silanol HOSi $\left(\mathrm{SiMe}_{3}\right)_{3}(3.00 \mathrm{~g}, 11.3 \mathrm{mmol})$ was mixed with $\mathrm{KH}(0.45 \mathrm{~g}, 11.2 \mathrm{mmol})$ and dry heptane $(15 \mathrm{~mL})$ in a reaction bulb equipped with a Kontes valve in an argon-filled drybox. After 1 day, sublimed $\mathrm{AlCl}_{3}(0.50 \mathrm{~g}$, $3.75 \mathrm{mmol}$ ) was added to the bulb with another $5 \mathrm{~mL}$ of heptane, and the bulb was sonicated for 1 day in a bath sonicator. The reaction was returned to the drybox and filtration was attempted through a fine frit. The frit clogged after a small amount of filtrate went through. Filtration was resumed through $1 \mathrm{~cm}$ diameter PTFE membranes with a nominal $0.22 \mu \mathrm{m}$ size. The membrane had to be changed twice, but filtration was finally completed after two weeks. Crystals of the title compound grew in the filtrate (64 mg isolated). The liquid was decanted from the crystals, the solvent removed, 
leaving a semi-solid mixture. An attempt at sublimation resulted in decomposition.

A repeat preparation using the same quantities of reactants in $40 \mathrm{~mL}$ heptane was sonicated for $3 \mathrm{~h}$ and then stirred at $338 \mathrm{~K}$ overnight. The mixture was filtered through a $47 \mathrm{~mm}$ diameter $0.22 \mu \mathrm{m}$ PVDF filter membrane, and the filtrate pumped to a white solid, whose NMR showed multiple products. This white solid was recrystallized from a minimum amount of hot heptane under argon, affording $1.335 \mathrm{~g} \mathrm{(40 \% )}$ of colorless crystals of the title compound. NMR $\left(\mathrm{C}_{6} \mathrm{D}_{6}\right):{ }^{1} \mathrm{H}$ 80.36; ${ }^{13} \mathrm{C} 3.36 ;{ }^{29} \mathrm{Si}-22.39(\mathrm{Si}),-18.36\left(\mathrm{SiMe}_{3}\right)$.

\section{Refinement}

Crystal data, data collection and structure refinement details are summarized in Table 1.

\section{Funding information}

Funding for this research was provided by: The Office of Naval Research .

\section{References}

Abdalla, J. A. B., Riddlestone, I. M., Tirfoin, R. \& Aldridge, S. (2015). Angew. Chem. Int. Ed. 54, 5098-5102.
Agilent (2014). CrysAlis PRO. Agilent Technologies Ltd, Yarnton, England.

Apblett, A. W., Warren, A. C. \& Barron, A. R. (1992). Can. J. Chem. 70, 771-778.

Boyle, T. J., Sears, J. M., Perales, D., Cramer, R. E., Lu, P., Chan, R. O. \& Hernandez-Sanchez, B. A. (2018). Inorg. Chem. 57, 8806-8820.

Chesnokova, T. A., Zhezlova, E. V., Kornev, A. N., Fedotova, Y. V., Zakharov, L. N., Fukin, G. K., Kursky, Y. A., Mushtina, T. G. \& Domrachev, G. A. (2002). J. Organomet. Chem. 642, 20-31.

Gilman, H. \& Harrell, R. L. (1966). J. Organomet. Chem. 5, 199-200.

Groom, C. R., Bruno, I. J., Lightfoot, M. P. \& Ward, S. C. (2016). Acta Cryst. B72, 171-179.

Kornev, N. A., Chesnokova, T. A., Semenov, V. V., Zhezlova, E. V., Zakharov, L. N., Klapshina, L. G., Domrachev, G. A. \& Rusakov, V. S. (1997). J. Organomet. Chem. 547, 113-119.

Kornev, N. A., Chesnokova, T. A., Zhezlova, E. V., Zakharov, L. N., Fukin, G. K., Kursky, Y. A., Domrachev, G. A. \& Lickiss, P. D. (1999). J. Organomet. Chem. 587, 113-121.

Krempner, C., Reinke, H. \& Weichert, K. (2007). Organometallics, 26, 1386-1392.

Leiner, E., Hampe, O. \& Scheer, M. (2002). Eur. J. Inorg. Chem. pp. 584-590.

Niemeyer, M. (2006). Inorg. Chem. 45, 9085-9095.

Sheldrick, G. M. (2008). Acta Cryst. A64, 112-122.

Sheldrick, G. M. (2015a). Acta Cryst. A71, 3-8.

Sheldrick, G. M. (2015b). Acta Cryst. C71, 3-8.

Wu, Z., Cai, H., Yu, X., Blanton, J. R., Diminnie, J. B., Pan, H., Xue, Z. \& Bryan, J. C. (2002). Organometallics, 21, 3973-3978. 


\section{supporting information}

Acta Cryst. (2019). E75, 714-716 [https://doi.org/10.1107/S2056989019005310]

\section{Potassium chloridotris(hypersiloxy)aluminate dimer}

\section{Andrew P. Purdy and Raymond J. Butcher}

\section{Computing details}

Data collection: CrysAlis PRO (Agilent, 2014); cell refinement: CrysAlis PRO (Agilent, 2014); data reduction: CrysAlis PRO (Agilent, 2014); program(s) used to solve structure: SHELXT (Sheldrick, 2015a); program(s) used to refine structure: SHELXL2016 (Sheldrick, 2015b); molecular graphics: SHELXTL (Sheldrick, 2008); software used to prepare material for publication: SHELXTL (Sheldrick, 2008).

Di- $\mu_{3}$-chlorido-bis[ $\mu_{2}$-tris(trimethylsilyl)silanolato] tetrakis[tris(trimethylsilyl)silanolato] dialuminiumdipotassium

Crystal data

$\left[\mathrm{Al}_{2} \mathrm{~K}_{2} \mathrm{Cl}_{2}\left(\mathrm{C}_{19} \mathrm{H}_{27} \mathrm{OSi}_{4}\right)_{6}\right]$

$M_{r}=1785.05$

Monoclinic, $P 2_{1} / n$

$a=18.8247(3) \AA$

$b=13.8850(2) \AA$

$c=22.2056(4) \AA$

$\beta=109.413(2)^{\circ}$

$V=5474.14(17) \AA^{3}$

$Z=2$

Data collection

Agilent Xcalibur Eos Gemini diffractometer

Radiation source: Enhance (Cu) X-ray Source

Detector resolution: 16.0416 pixels $\mathrm{mm}^{-1}$

$\omega$ scans

Absorption correction: multi-scan

(CrysAlis PRO; Agilent, 2014)

$T_{\min }=0.441, T_{\max }=1.000$

Refinement

Refinement on $F^{2}$

Least-squares matrix: full

$R\left[F^{2}>2 \sigma\left(F^{2}\right)\right]=0.042$

$w R\left(F^{2}\right)=0.117$

$S=1.04$

10450 reflections

433 parameters

0 restraints
$F(000)=1936$

$D_{\mathrm{x}}=1.083 \mathrm{Mg} \mathrm{m}^{-3}$

$\mathrm{Cu} K \alpha$ radiation, $\lambda=1.54184 \AA$

Cell parameters from 9927 reflections

$\theta=4.0-71.3^{\circ}$

$\mu=4.16 \mathrm{~mm}^{-1}$

$T=173 \mathrm{~K}$

Block, colorless

$0.37 \times 0.31 \times 0.24 \mathrm{~mm}$

25914 measured reflections 10450 independent reflections 8699 reflections with $I>2 \sigma(I)$

$R_{\text {int }}=0.035$

$\theta_{\text {max }}=71.4^{\circ}, \theta_{\text {min }}=3.8^{\circ}$

$h=-23 \rightarrow 19$

$k=-16 \rightarrow 16$

$l=-27 \rightarrow 25$

Hydrogen site location: inferred from neighbouring sites

$\mathrm{H}$-atom parameters constrained

$w=1 /\left[\sigma^{2}\left(F_{0}^{2}\right)+(0.0711 P)^{2}+0.624 P\right]$

where $P=\left(F_{\mathrm{o}}{ }^{2}+2 F_{\mathrm{c}}{ }^{2}\right) / 3$

$(\Delta / \sigma)_{\max }=0.001$

$\Delta \rho_{\max }=0.76 \mathrm{e}^{-3}$

$\Delta \rho_{\min }=-0.28$ e $\AA^{-3}$ 


\section{Special details}

Geometry. All esds (except the esd in the dihedral angle between two 1.s. planes) are estimated using the full covariance matrix. The cell esds are taken into account individually in the estimation of esds in distances, angles and torsion angles; correlations between esds in cell parameters are only used when they are defined by crystal symmetry. An approximate (isotropic) treatment of cell esds is used for estimating esds involving l.s. planes.

Fractional atomic coordinates and isotropic or equivalent isotropic displacement parameters $\left(\AA^{2}\right)$

\begin{tabular}{|c|c|c|c|c|}
\hline & $x$ & $y$ & $z$ & $U_{\text {iso }} * / U_{\text {eq }}$ \\
\hline K1 & $0.57420(3)$ & $0.37104(4)$ & $0.48201(3)$ & $0.04295(14)$ \\
\hline $\mathrm{Cl1}$ & $0.47952(3)$ & $0.53645(4)$ & $0.41286(3)$ & $0.03676(13)$ \\
\hline Sil & $0.73143(3)$ & $0.46759(4)$ & $0.44589(3)$ & $0.02465(12)$ \\
\hline $\mathrm{Si} 2$ & $0.57625(3)$ & $0.80409(4)$ & $0.38317(3)$ & $0.02508(13)$ \\
\hline $\mathrm{Si} 3$ & $0.47806(3)$ & $0.47751(4)$ & $0.23042(3)$ & $0.02570(13)$ \\
\hline Sil1 & $0.76601(4)$ & $0.30398(5)$ & $0.44632(3)$ & $0.03327(14)$ \\
\hline Si12 & $0.77130(4)$ & $0.51357(5)$ & $0.55459(3)$ & $0.03630(15)$ \\
\hline Si13 & $0.79768(3)$ & $0.55732(5)$ & $0.39166(3)$ & $0.03275(14)$ \\
\hline Si21 & $0.44683(3)$ & $0.83662(5)$ & $0.33310(3)$ & $0.03364(14)$ \\
\hline $\mathrm{Si} 22$ & $0.63846(4)$ & $0.88865(5)$ & $0.32264(4)$ & $0.04016(16)$ \\
\hline Si23 & $0.61445(4)$ & $0.86915(5)$ & $0.48644(3)$ & $0.03486(15)$ \\
\hline $\mathrm{Si} 31$ & $0.52190(4)$ & $0.31699(5)$ & $0.23980(3)$ & $0.03842(16)$ \\
\hline Si32 & $0.48618(3)$ & $0.54112(5)$ & $0.13426(3)$ & $0.03018(14)$ \\
\hline Si33 & $0.34999(4)$ & $0.46420(5)$ & $0.22239(3)$ & $0.03741(16)$ \\
\hline Al1 & $0.56972(3)$ & $0.56860(4)$ & $0.37091(3)$ & $0.02094(13)$ \\
\hline O1 & $0.63984(8)$ & $0.48937(10)$ & $0.41485(7)$ & $0.0261(3)$ \\
\hline $\mathrm{O} 2$ & $0.59216(8)$ & $0.68797(10)$ & $0.38410(7)$ & $0.0278(3)$ \\
\hline $\mathrm{O} 3$ & $0.53047(8)$ & $0.53867(11)$ & $0.29203(7)$ & $0.0288(3)$ \\
\hline C111 & $0.75888(18)$ & $0.2512(2)$ & $0.36675(14)$ & $0.0534(7)$ \\
\hline H11A & 0.780011 & 0.186011 & 0.372780 & $0.080^{*}$ \\
\hline H11B & 0.787022 & 0.291510 & 0.346281 & $0.080^{*}$ \\
\hline $\mathrm{H} 11 \mathrm{C}$ & 0.705870 & 0.248551 & 0.339614 & $0.080^{*}$ \\
\hline C112 & $0.71162(17)$ & $0.22111(19)$ & $0.48223(13)$ & $0.0451(6)$ \\
\hline H11D & 0.735733 & 0.157629 & 0.489608 & $0.068^{*}$ \\
\hline H11E & 0.659848 & 0.214767 & 0.452859 & $0.068^{*}$ \\
\hline $\mathrm{H} 11 \mathrm{~F}$ & 0.710990 & 0.247837 & 0.522896 & $0.068^{*}$ \\
\hline C113 & $0.86772(16)$ & $0.2993(2)$ & $0.49849(15)$ & $0.0540(7)$ \\
\hline $\mathrm{H} 11 \mathrm{G}$ & 0.884887 & 0.232238 & 0.503648 & $0.081^{*}$ \\
\hline $\mathrm{H} 11 \mathrm{H}$ & 0.873035 & 0.326784 & 0.540422 & $0.081^{*}$ \\
\hline H11I & 0.898232 & 0.336493 & 0.478592 & $0.081^{*}$ \\
\hline $\mathrm{C} 121$ & $0.76369(18)$ & $0.4170(2)$ & $0.61159(12)$ & $0.0530(7)$ \\
\hline $\mathrm{H} 12 \mathrm{~A}$ & 0.779474 & 0.443114 & 0.655056 & $0.080^{*}$ \\
\hline H12B & 0.796250 & 0.362763 & 0.609887 & $0.080^{*}$ \\
\hline $\mathrm{H} 12 \mathrm{C}$ & 0.711370 & 0.394934 & 0.599518 & $0.080^{*}$ \\
\hline $\mathrm{C} 122$ & $0.8713(2)$ & $0.5559(3)$ & $0.58315(15)$ & $0.0752(12)$ \\
\hline H12D & 0.884643 & 0.578207 & 0.627406 & $0.113^{*}$ \\
\hline H12E & 0.877071 & 0.609061 & 0.556098 & $0.113^{*}$ \\
\hline $\mathrm{H} 12 \mathrm{~F}$ & 0.904521 & 0.502731 & 0.580911 & $0.113^{*}$ \\
\hline $\mathrm{C} 123$ & $0.7030(3)$ & $0.6105(3)$ & $0.55869(17)$ & $0.0743(11)$ \\
\hline
\end{tabular}




\begin{tabular}{|c|c|c|c|c|}
\hline $\mathrm{H} 12 \mathrm{G}$ & 0.724758 & 0.648099 & 0.597890 & $0.111^{*}$ \\
\hline $\mathrm{H} 12 \mathrm{H}$ & 0.655816 & 0.580877 & 0.558750 & $0.111^{*}$ \\
\hline H12I & 0.693022 & 0.653051 & 0.521565 & $0.111^{*}$ \\
\hline $\mathrm{C} 131$ & $0.74560(17)$ & $0.5443(2)$ & $0.30394(12)$ & $0.0478(6)$ \\
\hline $\mathrm{H} 13 \mathrm{~A}$ & 0.767512 & 0.587768 & 0.280079 & $0.072 *$ \\
\hline H13B & 0.692443 & 0.560660 & 0.295038 & $0.072^{*}$ \\
\hline $\mathrm{H} 13 \mathrm{C}$ & 0.749622 & 0.477677 & 0.290865 & $0.072^{*}$ \\
\hline $\mathrm{C} 132$ & $0.79786(18)$ & $0.6873(2)$ & $0.41371(15)$ & $0.0513(7)$ \\
\hline H13D & 0.818651 & 0.725943 & 0.386573 & $0.077^{*}$ \\
\hline H13E & 0.828843 & 0.696002 & 0.458561 & $0.077^{*}$ \\
\hline $\mathrm{H} 13 \mathrm{~F}$ & 0.746187 & 0.708185 & 0.407553 & $0.077^{*}$ \\
\hline $\mathrm{C} 133$ & $0.89776(16)$ & $0.5175(3)$ & $0.40851(17)$ & $0.0598(8)$ \\
\hline H13G & 0.922440 & 0.560244 & 0.386404 & $0.090^{*}$ \\
\hline $\mathrm{H} 13 \mathrm{H}$ & 0.898381 & 0.451353 & 0.393329 & $0.090^{*}$ \\
\hline H13I & 0.924675 & 0.520185 & 0.454585 & $0.090^{*}$ \\
\hline C211 & $0.38795(14)$ & $0.77173(19)$ & $0.37411(14)$ & $0.0448(6)$ \\
\hline H21A & 0.334764 & 0.775116 & 0.347502 & $0.067^{*}$ \\
\hline $\mathrm{H} 21 \mathrm{~B}$ & 0.394737 & 0.802112 & 0.415485 & $0.067^{*}$ \\
\hline $\mathrm{H} 21 \mathrm{C}$ & 0.403673 & 0.704162 & 0.380699 & $0.067^{*}$ \\
\hline $\mathrm{C} 212$ & $0.42149(18)$ & $0.7911(2)$ & $0.24922(13)$ & $0.0543(7)$ \\
\hline $\mathrm{H} 21 \mathrm{D}$ & 0.367071 & 0.797614 & 0.227717 & $0.082^{*}$ \\
\hline $\mathrm{H} 21 \mathrm{E}$ & 0.435827 & 0.723211 & 0.249816 & $0.082^{*}$ \\
\hline $\mathrm{H} 21 \mathrm{~F}$ & 0.448313 & 0.828807 & 0.226233 & $0.082^{*}$ \\
\hline $\mathrm{C} 213$ & $0.41897(17)$ & $0.9676(2)$ & $0.33006(18)$ & $0.0574(8)$ \\
\hline $\mathrm{H} 21 \mathrm{G}$ & 0.367020 & 0.975242 & 0.301292 & $0.086^{*}$ \\
\hline $\mathrm{H} 21 \mathrm{H}$ & 0.452743 & 1.006107 & 0.314321 & $0.086^{*}$ \\
\hline H21I & 0.422672 & 0.989346 & 0.372975 & $0.086^{*}$ \\
\hline C221 & $0.6506(2)$ & $0.8050(2)$ & $0.26151(17)$ & $0.0658(9)$ \\
\hline $\mathrm{H} 22 \mathrm{~A}$ & 0.673766 & 0.839512 & 0.234291 & $0.099^{*}$ \\
\hline $\mathrm{H} 22 \mathrm{~B}$ & 0.601304 & 0.780109 & 0.235263 & $0.099^{*}$ \\
\hline $\mathrm{H} 22 \mathrm{C}$ & 0.683072 & 0.751315 & 0.282708 & $0.099 *$ \\
\hline C222 & $0.5826(2)$ & $0.9958(2)$ & $0.2800(2)$ & $0.0682(10)$ \\
\hline $\mathrm{H} 22 \mathrm{D}$ & 0.610576 & 1.028923 & 0.255949 & $0.102 *$ \\
\hline $\mathrm{H} 22 \mathrm{E}$ & 0.574274 & 1.040142 & 0.311312 & $0.102 *$ \\
\hline $\mathrm{H} 22 \mathrm{~F}$ & 0.533950 & 0.974001 & 0.250513 & $0.102 *$ \\
\hline $\mathrm{C} 223$ & $0.73237(18)$ & $0.9395(2)$ & $0.37176(19)$ & $0.0645(9)$ \\
\hline $\mathrm{H} 22 \mathrm{G}$ & 0.757250 & 0.967287 & 0.343418 & $0.097^{*}$ \\
\hline $\mathrm{H} 22 \mathrm{H}$ & 0.763844 & 0.888025 & 0.397332 & $0.097^{*}$ \\
\hline H22I & 0.724997 & 0.989669 & 0.400156 & $0.097 *$ \\
\hline C231 & $0.56436(17)$ & $0.8067(2)$ & $0.53611(13)$ & $0.0477(6)$ \\
\hline $\mathrm{H} 23 \mathrm{~A}$ & 0.584952 & 0.829141 & 0.580392 & $0.072 *$ \\
\hline H23B & 0.571624 & 0.736987 & 0.534685 & $0.072 *$ \\
\hline $\mathrm{H} 23 \mathrm{C}$ & 0.510450 & 0.821594 & 0.519109 & $0.072^{*}$ \\
\hline $\mathrm{C} 232$ & $0.5892(2)$ & 1.00077 (19) & $0.47939(16)$ & $0.0545(7)$ \\
\hline $\mathrm{H} 23 \mathrm{D}$ & 0.606557 & 1.030377 & 0.521865 & $0.082^{*}$ \\
\hline $\mathrm{H} 23 \mathrm{E}$ & 0.534465 & 1.007830 & 0.460654 & $0.082^{*}$ \\
\hline $\mathrm{H} 23 \mathrm{~F}$ & 0.613525 & 1.032639 & 0.451989 & $0.082 *$ \\
\hline C233 & $0.71955(18)$ & $0.8631(3)$ & $0.52913(16)$ & $0.0627(8)$ \\
\hline
\end{tabular}




$\begin{array}{lllll}\text { H23G } & 0.730299 & 0.874770 & 0.574827 & 0.094^{*} \\ \text { H23H } & 0.744673 & 0.912297 & 0.511712 & 0.094^{*} \\ \text { H23I } & 0.738178 & 0.799260 & 0.522965 & 0.094^{*} \\ \text { C311 } & 0.4504(2) & 0.2270(2) & 0.19217(15) & 0.0671(10) \\ \text { H31A } & 0.474201 & 0.163627 & 0.194905 & 0.101^{*} \\ \text { H31B } & 0.430846 & 0.247478 & 0.147429 & 0.101^{*} \\ \text { H31C } & 0.408789 & 0.222938 & 0.209386 & 0.101^{*} \\ \text { C312 } & 0.6072(2) & 0.3029(2) & 0.21447(15) & 0.0596(8) \\ \text { H31D } & 0.628708 & 0.238580 & 0.226263 & 0.089^{*} \\ \text { H31E } & 0.644708 & 0.351717 & 0.235836 & 0.089^{*} \\ \text { H31F } & 0.592593 & 0.311132 & 0.168107 & 0.089^{*} \\ \text { C313 } & 0.54652(17) & 0.28382(19) & 0.32684(12) & 0.0446(6) \\ \text { H31G } & 0.564946 & 0.217323 & 0.333273 & 0.067^{*} \\ \text { H31H } & 0.501596 & 0.289644 & 0.339574 & 0.067^{*} \\ \text { H31I } & 0.585781 & 0.327295 & 0.352851 & 0.067^{*} \\ \text { C321 } & 0.4486(2) & 0.4501(2) & 0.06848(13) & 0.0594(8) \\ \text { H32A } & 0.455440 & 0.474287 & 0.029288 & 0.089^{*} \\ \text { H32B } & 0.394937 & 0.439418 & 0.061008 & 0.089^{*} \\ \text { H32C } & 0.476025 & 0.389309 & 0.080909 & 0.089^{*} \\ \text { C322 } & 0.43252(19) & 0.6563(2) & 0.10763(14) & 0.0564(7) \\ \text { H32D } & 0.436173 & 0.675891 & 0.066364 & 0.085^{*} \\ \text { H32E } & 0.453887 & 0.706765 & 0.139331 & 0.085^{*} \\ \text { H32F } & 0.379520 & 0.646261 & 0.103305 & 0.085^{*} \\ \text { C323 } & 0.58614(15) & 0.5661(3) & 0.14213(14) & 0.0519(7) \\ \text { H32G } & 0.588707 & 0.589139 & 0.101178 & 0.078^{*} \\ \text { H32H } & 0.615790 & 0.506953 & 0.154461 & 0.078^{*} \\ \text { H32I } & 0.606491 & 0.615507 & 0.174890 & 0.078^{*} \\ \text { C331 } & 0.33467(19) & 0.3778(3) & 0.28202(14) & 0.0657(10) \\ \text { H33A } & 0.280583 & 0.371597 & 0.274680 & 0.099^{*} \\ \text { H33B } & 0.360012 & 0.402047 & 0.325314 & 0.099^{*} \\ \text { H33C } & 0.355427 & 0.314680 & 0.277160 & 0.099^{*} \\ \text { C332 } & 0.29642(15) & 0.4164(2) & 0.14070(12) & 0.0471(6) \\ \text { H33D } & 0.242748 & 0.412544 & 0.135569 & 0.071^{*} \\ \text { H33E } & 0.315193 & 0.352144 & 0.135671 & 0.071^{*} \\ \text { H33F } & 0.303400 & 0.459714 & 0.108249 & 0.071^{*} \\ \text { C333 } & 0.30521(16) & 0.5810(3) & 0.23174(17) & 0.0604(8) \\ \text { H33G } & 0.252048 & 0.570193 & 0.226489 & 0.091^{*} \\ \text { H33H } & 0.309020 & 0.626685 & 0.199285 & 0.091^{*} \\ \text { H33I } & 0.331125 & 0.607448 & 0.274338 & 0.091^{*} \\ & & & & \end{array}$

Atomic displacement parameters $\left(\AA^{2}\right)$

\begin{tabular}{lllllll}
\hline & $U^{11}$ & $U^{22}$ & $U^{\beta 3}$ & $U^{12}$ & $U^{13}$ & $U^{23}$ \\
\hline K1 & $0.0524(3)$ & $0.0352(3)$ & $0.0494(3)$ & $0.0059(2)$ & $0.0278(3)$ & $0.0117(2)$ \\
C11 & $0.0353(3)$ & $0.0404(3)$ & $0.0420(3)$ & $0.0060(2)$ & $0.0228(2)$ & $0.0082(2)$ \\
Si1 & $0.0240(3)$ & $0.0245(3)$ & $0.0269(3)$ & $0.0041(2)$ & $0.0104(2)$ & $0.0041(2)$ \\
Si2 & $0.0270(3)$ & $0.0184(3)$ & $0.0336(3)$ & $-0.0010(2)$ & $0.0153(2)$ & $-0.0031(2)$ \\
Si3 & $0.0269(3)$ & $0.0277(3)$ & $0.0226(3)$ & $-0.0050(2)$ & $0.0083(2)$ & $-0.0025(2)$
\end{tabular}




\begin{tabular}{|c|c|c|c|c|c|c|}
\hline Si11 & $0.0381(3)$ & $0.0288(3)$ & $0.0358(3)$ & $0.0127(2)$ & $0.0162(3)$ & $0.0085(2)$ \\
\hline $\operatorname{Si} 12$ & $0.0408(3)$ & $0.0391(4)$ & $0.0291(3)$ & $0.0003(3)$ & $0.0117(3)$ & $-0.0002(3)$ \\
\hline $\operatorname{Si13}$ & $0.0298(3)$ & $0.0355(3)$ & $0.0379(3)$ & $0.0023(2)$ & $0.0178(2)$ & $0.0055(3)$ \\
\hline Si21 & $0.0303(3)$ & $0.0282(3)$ & $0.0436(3)$ & $0.0052(2)$ & $0.0138(3)$ & $0.0051(3)$ \\
\hline $\mathrm{Si} 22$ & $0.0499(4)$ & $0.0252(3)$ & $0.0597(4)$ & 0.0017 (3) & $0.0375(3)$ & $0.0051(3)$ \\
\hline Si23 & $0.0404(3)$ & $0.0271(3)$ & $0.0401(3)$ & $-0.0048(2)$ & $0.0175(3)$ & $-0.0115(3)$ \\
\hline $\mathrm{Si} 31$ & $0.0528(4)$ & $0.0270(3)$ & $0.0310(3)$ & $-0.0010(3)$ & $0.0079(3)$ & $-0.0054(2)$ \\
\hline Si32 & $0.0312(3)$ & $0.0366(3)$ & $0.0236(3)$ & $-0.0053(2)$ & $0.0103(2)$ & $-0.0019(2)$ \\
\hline Si33 & $0.0304(3)$ & $0.0495(4)$ & $0.0332(3)$ & $-0.0151(3)$ & $0.0118(2)$ & $-0.0052(3)$ \\
\hline Al1 & $0.0223(3)$ & $0.0180(3)$ & $0.0226(3)$ & $0.0010(2)$ & $0.0075(2)$ & $-0.0008(2)$ \\
\hline $\mathrm{O} 1$ & $0.0240(7)$ & $0.0234(7)$ & $0.0308(7)$ & $0.0039(5)$ & $0.0088(6)$ & $0.0026(6)$ \\
\hline $\mathrm{O} 2$ & $0.0302(7)$ & $0.0190(7)$ & $0.0343(7)$ & $-0.0003(6)$ & $0.0108(6)$ & $-0.0037(6)$ \\
\hline $\mathrm{O} 3$ & $0.0320(7)$ & $0.0294(8)$ & $0.0238(7)$ & $-0.0048(6)$ & $0.0075(6)$ & $-0.0048(6)$ \\
\hline C111 & $0.0675(18)$ & $0.0499(17)$ & $0.0515(15)$ & $0.0184(14)$ & $0.0315(14)$ & $-0.0021(13)$ \\
\hline $\mathrm{C} 112$ & $0.0609(16)$ & $0.0307(13)$ & $0.0491(14)$ & $0.0054(11)$ & $0.0257(12)$ & $0.0119(11)$ \\
\hline $\mathrm{C} 113$ & $0.0431(14)$ & $0.0533(17)$ & $0.0627(17)$ & $0.0198(13)$ & $0.0135(13)$ & $0.0171(14)$ \\
\hline $\mathrm{C} 121$ & $0.0633(17)$ & $0.0625(19)$ & $0.0324(12)$ & $0.0003(15)$ & $0.0146(12)$ & $0.0096(13)$ \\
\hline $\mathrm{C} 122$ & $0.065(2)$ & $0.107(3)$ & $0.0441(16)$ & $-0.042(2)$ & $0.0053(15)$ & $-0.0160(18)$ \\
\hline $\mathrm{C} 123$ & 0.118 & $0.058(2)$ & $0.0582(19)$ & $0.032(2)$ & $0.045(2)$ & $0.0017(16)$ \\
\hline $\mathrm{C} 131$ & $0.0582(16)$ & $0.0527(16)$ & $0.0373(13)$ & $0.0033(13)$ & $0.0224(12)$ & $0.0050(12)$ \\
\hline $\mathrm{C} 132$ & $0.0608(17)$ & $0.0366(14)$ & $0.0649(17)$ & $-0.0077(12)$ & $0.0322(14)$ & $0.0028(13)$ \\
\hline $\mathrm{C} 133$ & $0.0366(14)$ & $0.076(2)$ & $0.075(2)$ & $0.0113(14)$ & $0.0303(14)$ & $0.0167(17)$ \\
\hline $\mathrm{C} 211$ & $0.0378(12)$ & $0.0379(14)$ & $0.0663(17)$ & $0.0050(10)$ & $0.0275(12)$ & $0.0052(12)$ \\
\hline $\mathrm{C} 212$ & $0.0565(16)$ & $0.0589(18)$ & 0.0407 (14) & $-0.0038(14)$ & $0.0069(12)$ & $0.0012(13)$ \\
\hline $\mathrm{C} 213$ & $0.0492(16)$ & $0.0353(15)$ & $0.090(2)$ & $0.0140(12)$ & $0.0258(16)$ & $0.0115(14)$ \\
\hline $\mathrm{C} 221$ & $0.099(3)$ & $0.0534(19)$ & $0.070(2)$ & $0.0097(17)$ & $0.061(2)$ & $0.0019(16)$ \\
\hline $\mathrm{C} 222$ & $0.081(2)$ & $0.0392(16)$ & $0.105(3)$ & $0.0161(15)$ & $0.058(2)$ & $0.0314(17)$ \\
\hline $\mathrm{C} 223$ & $0.0545(17)$ & $0.0474(17)$ & $0.105(3)$ & $-0.0157(14)$ & $0.0445(18)$ & $0.0011(17)$ \\
\hline $\mathrm{C} 231$ & $0.0620(16)$ & $0.0406(14)$ & $0.0496(14)$ & $0.0032(12)$ & $0.0305(13)$ & $-0.0003(12)$ \\
\hline $\mathrm{C} 232$ & $0.078(2)$ & $0.0283(13)$ & $0.0685(18)$ & $-0.0056(13)$ & $0.0391(16)$ & $-0.0143(13)$ \\
\hline $\mathrm{C} 233$ & $0.0481(16)$ & $0.066(2)$ & $0.0626(19)$ & $-0.0140(15)$ & $0.0029(14)$ & $-0.0167(16)$ \\
\hline $\mathrm{C} 311$ & $0.093(3)$ & $0.0316(14)$ & $0.0540(17)$ & $-0.0107(15)$ & $-0.0057(16)$ & $-0.0135(13)$ \\
\hline $\mathrm{C} 312$ & $0.077(2)$ & $0.0561(19)$ & $0.0505(16)$ & $0.0246(16)$ & $0.0274(15)$ & $0.0020(14)$ \\
\hline $\mathrm{C} 313$ & $0.0589(16)$ & $0.0329(13)$ & $0.0356(12)$ & $-0.0020(11)$ & $0.0071(11)$ & $0.0028(10)$ \\
\hline $\mathrm{C} 321$ & $0.080(2)$ & $0.066(2)$ & $0.0346(13)$ & $-0.0285(17)$ & $0.0223(14)$ & $-0.0183(13)$ \\
\hline $\mathrm{C} 322$ & $0.0623(18)$ & $0.0596(19)$ & $0.0481(15)$ & $0.0176(15)$ & $0.0195(14)$ & $0.0193(14)$ \\
\hline $\mathrm{C} 323$ & $0.0360(13)$ & $0.076(2)$ & $0.0490(15)$ & $-0.0073(13)$ & $0.0212(11)$ & $0.0049(14)$ \\
\hline $\mathrm{C} 331$ & $0.0564(17)$ & $0.097(3)$ & $0.0460(15)$ & $-0.0347(18)$ & $0.0200(13)$ & $0.0073(16)$ \\
\hline C332 & $0.0406(13)$ & $0.0558(17)$ & $0.0375(12)$ & $-0.0154(12)$ & $0.0033(10)$ & $-0.0077(12)$ \\
\hline C333 & $0.0378(14)$ & $0.071(2)$ & $0.077(2)$ & $-0.0106(14)$ & $0.0254(14)$ & $-0.0253(17)$ \\
\hline
\end{tabular}

Geometric parameters $\left(\AA,{ }^{\circ}\right)$

\begin{tabular}{llll}
\hline $\mathrm{K} 1-\mathrm{O} 1$ & $2.7703(16)$ & $\mathrm{C} 123-\mathrm{H} 12 \mathrm{G}$ & 0.9800 \\
$\mathrm{~K} 1-\mathrm{C} 11$ & $2.9984(8)$ & $\mathrm{C} 123-\mathrm{H} 12 \mathrm{H}$ & 0.9800 \\
$\mathrm{~K} 1-\mathrm{C} 11^{\mathrm{i}}$ & $3.1131(8)$ & $\mathrm{C} 123-\mathrm{H} 12 \mathrm{I}$ & 0.9800 \\
$\mathrm{~K} 1-\mathrm{C} 112$ & $3.319(3)$ & $\mathrm{C} 131-\mathrm{H} 13 \mathrm{~A}$ & 0.9800 \\
$\mathrm{~K} 1-\mathrm{C} 231^{\mathrm{i}}$ & $3.516(3)$ & $\mathrm{C} 131-\mathrm{H} 13 \mathrm{~B}$ & 0.9800
\end{tabular}




\begin{tabular}{|c|c|c|c|}
\hline $\mathrm{K} 1-\mathrm{C} 313$ & $3.524(3)$ & $\mathrm{C} 131-\mathrm{H} 13 \mathrm{C}$ & 0.9800 \\
\hline $\mathrm{K} 1-\mathrm{Si} 1$ & $3.5747(8)$ & C132-H13D & 0.9800 \\
\hline $\mathrm{K} 1-\mathrm{A} 11$ & $3.6714(8)$ & C132-H13E & 0.9800 \\
\hline $\mathrm{K} 1-\mathrm{K} 1^{\mathrm{i}}$ & $4.7699(11)$ & C132-H13F & 0.9800 \\
\hline $\mathrm{C} 11-\mathrm{A} 11$ & $2.2366(7)$ & $\mathrm{C} 133-\mathrm{H} 13 \mathrm{G}$ & 0.9800 \\
\hline $\mathrm{Si} 1-\mathrm{O} 1$ & $1.6581(14)$ & $\mathrm{C} 133-\mathrm{H} 13 \mathrm{H}$ & 0.9800 \\
\hline Si1-Si13 & $2.3573(8)$ & C133-H13I & 0.9800 \\
\hline Sil-Si11 & $2.3623(8)$ & $\mathrm{C} 211-\mathrm{H} 21 \mathrm{~A}$ & 0.9800 \\
\hline Si1-Si12 & $2.3648(8)$ & $\mathrm{C} 211-\mathrm{H} 21 \mathrm{~B}$ & 0.9800 \\
\hline $\mathrm{Si} 2-\mathrm{O} 2$ & $1.6387(15)$ & $\mathrm{C} 211-\mathrm{H} 21 \mathrm{C}$ & 0.9800 \\
\hline $\mathrm{Si} 2-\mathrm{Si} 23$ & $2.3443(8)$ & $\mathrm{C} 212-\mathrm{H} 21 \mathrm{D}$ & 0.9800 \\
\hline $\mathrm{Si} 2-\mathrm{Si} 21$ & $2.3612(8)$ & $\mathrm{C} 212-\mathrm{H} 21 \mathrm{E}$ & 0.9800 \\
\hline $\mathrm{Si} 2-\mathrm{Si} 22$ & $2.3670(8)$ & $\mathrm{C} 212-\mathrm{H} 21 \mathrm{~F}$ & 0.9800 \\
\hline $\mathrm{Si} 3-\mathrm{O} 3$ & $1.6337(15)$ & $\mathrm{C} 213-\mathrm{H} 21 \mathrm{G}$ & 0.9800 \\
\hline $\mathrm{Si} 3-\mathrm{Si} 31$ & $2.3617(9)$ & $\mathrm{C} 213-\mathrm{H} 21 \mathrm{H}$ & 0.9800 \\
\hline $\mathrm{Si} 3-\mathrm{Si} 32$ & $2.3623(8)$ & $\mathrm{C} 213-\mathrm{H} 21 \mathrm{I}$ & 0.9800 \\
\hline $\mathrm{Si} 3-\mathrm{Si} 33$ & $2.3648(8)$ & $\mathrm{C} 221-\mathrm{H} 22 \mathrm{~A}$ & 0.9800 \\
\hline Si11-C111 & $1.876(3)$ & $\mathrm{C} 221-\mathrm{H} 22 \mathrm{~B}$ & 0.9800 \\
\hline Si11-C113 & $1.881(3)$ & $\mathrm{C} 221-\mathrm{H} 22 \mathrm{C}$ & 0.9800 \\
\hline Si11-C112 & $1.884(3)$ & C222-H22D & 0.9800 \\
\hline $\mathrm{Si} 12-\mathrm{C} 122$ & $1.870(3)$ & $\mathrm{C} 222-\mathrm{H} 22 \mathrm{E}$ & 0.9800 \\
\hline Si12-C121 & $1.882(3)$ & $\mathrm{C} 222-\mathrm{H} 22 \mathrm{~F}$ & 0.9800 \\
\hline Si12-C123 & $1.884(3)$ & $\mathrm{C} 223-\mathrm{H} 22 \mathrm{G}$ & 0.9800 \\
\hline Si13-C132 & $1.870(3)$ & $\mathrm{C} 223-\mathrm{H} 22 \mathrm{H}$ & 0.9800 \\
\hline Si13-C131 & $1.876(3)$ & $\mathrm{C} 223-\mathrm{H} 22 \mathrm{I}$ & 0.9800 \\
\hline Si13-C133 & $1.878(3)$ & $\mathrm{C} 231-\mathrm{H} 23 \mathrm{~A}$ & 0.9800 \\
\hline Si21-C212 & $1.872(3)$ & $\mathrm{C} 231-\mathrm{H} 23 \mathrm{~B}$ & 0.9800 \\
\hline Si21-C211 & $1.882(3)$ & $\mathrm{C} 231-\mathrm{H} 23 \mathrm{C}$ & 0.9800 \\
\hline Si21-C213 & $1.887(3)$ & $\mathrm{C} 232-\mathrm{H} 23 \mathrm{D}$ & 0.9800 \\
\hline $\mathrm{Si} 22-\mathrm{C} 221$ & $1.857(3)$ & $\mathrm{C} 232-\mathrm{H} 23 \mathrm{E}$ & 0.9800 \\
\hline $\mathrm{Si} 22-\mathrm{C} 223$ & $1.879(3)$ & $\mathrm{C} 232-\mathrm{H} 23 \mathrm{~F}$ & 0.9800 \\
\hline $\mathrm{Si} 22-\mathrm{C} 222$ & $1.885(3)$ & $\mathrm{C} 233-\mathrm{H} 23 \mathrm{G}$ & 0.9800 \\
\hline $\mathrm{Si} 23-\mathrm{C} 232$ & $1.882(3)$ & $\mathrm{C} 233-\mathrm{H} 23 \mathrm{H}$ & 0.9800 \\
\hline Si23-C231 & $1.884(3)$ & $\mathrm{C} 233-\mathrm{H} 23 \mathrm{I}$ & 0.9800 \\
\hline Si23-C233 & $1.890(3)$ & C311-H31A & 0.9800 \\
\hline Si31-C312 & $1.879(3)$ & C311-H31B & 0.9800 \\
\hline Si31-C311 & $1.884(3)$ & $\mathrm{C} 311-\mathrm{H} 31 \mathrm{C}$ & 0.9800 \\
\hline Si31-C313 & $1.889(3)$ & C312-H31D & 0.9800 \\
\hline $\mathrm{Si} 32-\mathrm{C} 323$ & $1.864(3)$ & C312-H31E & 0.9800 \\
\hline $\mathrm{Si} 32-\mathrm{C} 322$ & $1.879(3)$ & $\mathrm{C} 312-\mathrm{H} 31 \mathrm{~F}$ & 0.9800 \\
\hline $\mathrm{Si} 32-\mathrm{C} 321$ & $1.882(3)$ & C313-H31G & 0.9800 \\
\hline Si33-C333 & $1.871(3)$ & $\mathrm{C} 313-\mathrm{H} 31 \mathrm{H}$ & 0.9800 \\
\hline Si33-C331 & $1.879(3)$ & C313-H31I & 0.9800 \\
\hline Si33-C332 & $1.879(2)$ & C321-H32A & 0.9800 \\
\hline $\mathrm{A} 11-\mathrm{O} 3$ & $1.7107(14)$ & C $321-\mathrm{H} 32 \mathrm{~B}$ & 0.9800 \\
\hline $\mathrm{A} 11-\mathrm{O} 2$ & $1.7115(15)$ & C $321-\mathrm{H} 32 \mathrm{C}$ & 0.9800 \\
\hline $\mathrm{A} 11-\mathrm{O} 1$ & $1.7459(15)$ & $\mathrm{C} 322-\mathrm{H} 32 \mathrm{D}$ & 0.9800 \\
\hline C111-H11A & 0.9800 & C322-H32E & 0.9800 \\
\hline
\end{tabular}




\begin{tabular}{|c|c|c|c|}
\hline C111-H11B & 0.9800 & $\mathrm{C} 322-\mathrm{H} 32 \mathrm{~F}$ & 0.9800 \\
\hline C111-H11C & 0.9800 & $\mathrm{C} 323-\mathrm{H} 32 \mathrm{G}$ & 0.9800 \\
\hline C112-H11D & 0.9800 & $\mathrm{C} 323-\mathrm{H} 32 \mathrm{H}$ & 0.9800 \\
\hline $\mathrm{C} 112-\mathrm{H} 11 \mathrm{E}$ & 0.9800 & $\mathrm{C} 323-\mathrm{H} 32 \mathrm{I}$ & 0.9800 \\
\hline $\mathrm{C} 112-\mathrm{H} 11 \mathrm{~F}$ & 0.9800 & $\mathrm{C} 331-\mathrm{H} 33 \mathrm{~A}$ & 0.9800 \\
\hline $\mathrm{C} 113-\mathrm{H} 11 \mathrm{G}$ & 0.9800 & C331-H33B & 0.9800 \\
\hline $\mathrm{C} 113-\mathrm{H} 11 \mathrm{H}$ & 0.9800 & $\mathrm{C} 331-\mathrm{H} 33 \mathrm{C}$ & 0.9800 \\
\hline C113-H11I & 0.9800 & C332-H33D & 0.9800 \\
\hline $\mathrm{C} 121-\mathrm{H} 12 \mathrm{~A}$ & 0.9800 & $\mathrm{C} 332-\mathrm{H} 33 \mathrm{E}$ & 0.9800 \\
\hline C121-H12B & 0.9800 & $\mathrm{C} 332-\mathrm{H} 33 \mathrm{~F}$ & 0.9800 \\
\hline $\mathrm{C} 121-\mathrm{H} 12 \mathrm{C}$ & 0.9800 & $\mathrm{C} 333-\mathrm{H} 33 \mathrm{G}$ & 0.9800 \\
\hline $\mathrm{C} 122-\mathrm{H} 12 \mathrm{D}$ & 0.9800 & $\mathrm{C} 333-\mathrm{H} 33 \mathrm{H}$ & 0.9800 \\
\hline $\mathrm{C} 122-\mathrm{H} 12 \mathrm{E}$ & 0.9800 & C $333-\mathrm{H} 33 \mathrm{I}$ & 0.9800 \\
\hline $\mathrm{C} 122-\mathrm{H} 12 \mathrm{~F}$ & 0.9800 & & \\
\hline $\mathrm{O} 1-\mathrm{K} 1-\mathrm{Cl1}$ & $64.26(3)$ & $\mathrm{Si} 12-\mathrm{C} 121-\mathrm{H} 12 \mathrm{~A}$ & 109.5 \\
\hline $\mathrm{O} 1-\mathrm{K} 1-\mathrm{C} 11^{\mathrm{i}}$ & 118.23 & $\mathrm{Si} 12-\mathrm{C} 121-\mathrm{H} 12 \mathrm{~B}$ & 109.5 \\
\hline $\mathrm{C} 11-\mathrm{K} 1-\mathrm{C} 11^{\mathrm{i}}$ & $77.41(2)$ & $\mathrm{H} 12 \mathrm{~A}-\mathrm{C} 121-\mathrm{H} 12 \mathrm{~B}$ & 109.5 \\
\hline $\mathrm{O} 1-\mathrm{K} 1-\mathrm{C} 112$ & $83.45(5)$ & $\mathrm{Si} 12-\mathrm{C} 121-\mathrm{H} 12 \mathrm{C}$ & 109.5 \\
\hline $\mathrm{C} 11-\mathrm{K} 1-\mathrm{C} 112$ & $144.25(5)$ & $\mathrm{H} 12 \mathrm{~A}-\mathrm{C} 121-\mathrm{H} 12 \mathrm{C}$ & 109.5 \\
\hline $\mathrm{C} 11^{\mathrm{i}-\mathrm{K} 1-\mathrm{C} 112}$ & $134.82(5)$ & $\mathrm{H} 12 \mathrm{~B}-\mathrm{C} 121-\mathrm{H} 12 \mathrm{C}$ & 109.5 \\
\hline $\mathrm{O} 1-\mathrm{K} 1-\mathrm{C} 231^{\mathrm{i}}$ & $143.27(6)$ & $\mathrm{Si} 12-\mathrm{C} 122-\mathrm{H} 12 \mathrm{D}$ & 109.5 \\
\hline $\mathrm{C} 11-\mathrm{K} 1-\mathrm{C} 231^{\mathrm{i}}$ & $100.78(5)$ & $\mathrm{Si} 12-\mathrm{C} 122-\mathrm{H} 12 \mathrm{E}$ & 109.5 \\
\hline $\mathrm{C} 11^{\mathrm{i}}-\mathrm{K} 1-\mathrm{C} 231^{\mathrm{i}}$ & $87.82(5)$ & $\mathrm{H} 12 \mathrm{D}-\mathrm{C} 122-\mathrm{H} 12 \mathrm{E}$ & 109.5 \\
\hline $\mathrm{C} 112-\mathrm{K} 1-\mathrm{C} 231^{\mathrm{i}}$ & $96.20(7)$ & $\mathrm{Si} 12-\mathrm{C} 122-\mathrm{H} 12 \mathrm{~F}$ & 109.5 \\
\hline $\mathrm{O} 1-\mathrm{K} 1-\mathrm{C} 313$ & $67.97(6)$ & $\mathrm{H} 12 \mathrm{D}-\mathrm{C} 122-\mathrm{H} 12 \mathrm{~F}$ & 109.5 \\
\hline $\mathrm{C} 11-\mathrm{K} 1-\mathrm{C} 313$ & $83.86(5)$ & $\mathrm{H} 12 \mathrm{E}-\mathrm{C} 122-\mathrm{H} 12 \mathrm{~F}$ & 109.5 \\
\hline 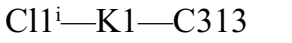 & $153.60(5)$ & $\mathrm{Si} 12-\mathrm{C} 123-\mathrm{H} 12 \mathrm{G}$ & 109.5 \\
\hline $\mathrm{C} 112-\mathrm{K} 1-\mathrm{C} 313$ & $69.38(7)$ & $\mathrm{Si} 12-\mathrm{C} 123-\mathrm{H} 12 \mathrm{H}$ & 109.5 \\
\hline $\mathrm{C} 231^{\mathrm{i}}-\mathrm{K} 1-\mathrm{C} 313$ & $77.51(7)$ & $\mathrm{H} 12 \mathrm{G}-\mathrm{C} 123-\mathrm{H} 12 \mathrm{H}$ & 109.5 \\
\hline $\mathrm{O} 1-\mathrm{K} 1-\mathrm{Si} 1$ & $26.64(3)$ & $\mathrm{Si} 12-\mathrm{C} 123-\mathrm{H} 12 \mathrm{I}$ & 109.5 \\
\hline $\mathrm{Cl1}-\mathrm{K} 1-\mathrm{Si} 1$ & $89.626(18)$ & $\mathrm{H} 12 \mathrm{G}-\mathrm{C} 123-\mathrm{H} 12 \mathrm{I}$ & 109.5 \\
\hline $\mathrm{C} 11^{\mathrm{i}}-\mathrm{K} 1-\mathrm{Si} 1$ & $120.87(2)$ & $\mathrm{H} 12 \mathrm{H}-\mathrm{C} 123-\mathrm{H} 12 \mathrm{I}$ & 109.5 \\
\hline $\mathrm{C} 112-\mathrm{K} 1-\mathrm{Si} 1$ & $62.13(5)$ & Si13-C131-H13A & 109.5 \\
\hline 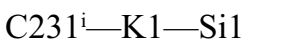 & $151.13(5)$ & Si13-C131-H13B & 109.5 \\
\hline C $313-\mathrm{K} 1-\mathrm{Si} 1$ & $76.93(5)$ & $\mathrm{H} 13 \mathrm{~A}-\mathrm{C} 131-\mathrm{H} 13 \mathrm{~B}$ & 109.5 \\
\hline $\mathrm{O} 1-\mathrm{K} 1-\mathrm{A} 11$ & $27.12(3)$ & Si13-C131-H13C & 109.5 \\
\hline $\mathrm{C} 11-\mathrm{K} 1-\mathrm{A} 11$ & $37.498(14)$ & $\mathrm{H} 13 \mathrm{~A}-\mathrm{C} 131-\mathrm{H} 13 \mathrm{C}$ & 109.5 \\
\hline 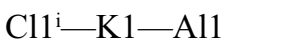 & $104.77(2)$ & $\mathrm{H} 13 \mathrm{~B}-\mathrm{C} 131-\mathrm{H} 13 \mathrm{C}$ & 109.5 \\
\hline $\mathrm{C} 112-\mathrm{K} 1-\mathrm{A} 11$ & $108.27(5)$ & Si13-C132-H13D & 109.5 \\
\hline 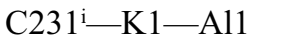 & $126.67(5)$ & $\mathrm{Si} 13-\mathrm{C} 132-\mathrm{H} 13 \mathrm{E}$ & 109.5 \\
\hline C313-K1-Al1 & $68.71(4)$ & $\mathrm{H} 13 \mathrm{D}-\mathrm{C} 132-\mathrm{H} 13 \mathrm{E}$ & 109.5 \\
\hline Si1-K1-Al1 & $53.478(14)$ & $\mathrm{Si} 13-\mathrm{C} 132-\mathrm{H} 13 \mathrm{~F}$ & 109.5 \\
\hline $\mathrm{O} 1-\mathrm{K} 1-\mathrm{K} 1^{\mathrm{i}}$ & $92.05(3)$ & $\mathrm{H} 13 \mathrm{D}-\mathrm{C} 132-\mathrm{H} 13 \mathrm{~F}$ & 109.5 \\
\hline $\mathrm{C} 11-\mathrm{K} 1-\mathrm{K} 1^{\mathrm{i}}$ & $39.564(13)$ & $\mathrm{H} 13 \mathrm{E}-\mathrm{C} 132-\mathrm{H} 13 \mathrm{~F}$ & 109.5 \\
\hline $\mathrm{C} 11^{\mathrm{i}}-\mathrm{K} 1-\mathrm{K} 1^{\mathrm{i}}$ & $37.842(13)$ & Si13-C133-H13G & 109.5 \\
\hline $\mathrm{C} 112-\mathrm{K} 1-\mathrm{K} 1^{\mathrm{i}}$ & $165.98(6)$ & $\mathrm{Si13}-\mathrm{C} 133-\mathrm{H} 13 \mathrm{H}$ & 109.5 \\
\hline 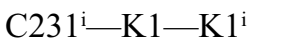 & $95.32(5)$ & $\mathrm{H} 13 \mathrm{G}-\mathrm{C} 133-\mathrm{H} 13 \mathrm{H}$ & 109.5 \\
\hline
\end{tabular}




\begin{tabular}{|c|c|}
\hline $\mathrm{C} 313-\mathrm{K} 1-\mathrm{K} 1^{\mathrm{i}}$ & $121.15(5)$ \\
\hline $\mathrm{Si} 1-\mathrm{K} 1-\mathrm{K} 1^{\mathrm{i}}$ & $109.31(2)$ \\
\hline $\mathrm{A} 11-\mathrm{K} 1-\mathrm{K} 1^{\mathrm{i}}$ & $70.590(15)$ \\
\hline $\mathrm{A} 11-\mathrm{C} 11-\mathrm{K} 1$ & $87.81(2)$ \\
\hline $\mathrm{A} 11-\mathrm{C} 11-\mathrm{K} 1^{\mathrm{i}}$ & $135.30(3)$ \\
\hline $\mathrm{K} 1-\mathrm{C} 11-\mathrm{K} 1^{\mathrm{i}}$ & $102.60(2)$ \\
\hline $\mathrm{O} 1-\mathrm{Si} 1-\mathrm{Si} 13$ & $110.18(6)$ \\
\hline $\mathrm{O} 1-\mathrm{Si} 1-\mathrm{Si} 11$ & $115.10(6)$ \\
\hline Si13-Si1-Si11 & $108.57(3)$ \\
\hline $\mathrm{O} 1-\mathrm{Si} 1-\mathrm{Si} 12$ & $107.96(6)$ \\
\hline Si13-Si1-Si12 & $109.66(3)$ \\
\hline Si11-Si1—Si12 & $105.19(3)$ \\
\hline $\mathrm{O} 1-\mathrm{Si} 1-\mathrm{K} 1$ & $48.51(5)$ \\
\hline Si13-Si1-K1 & $158.58(3)$ \\
\hline Si11-Si1-K1 & $83.46(2)$ \\
\hline Si12-Si1-K1 & $82.98(2)$ \\
\hline $\mathrm{O} 2-\mathrm{Si} 2-\mathrm{Si} 23$ & $111.81(6)$ \\
\hline $\mathrm{O} 2-\mathrm{Si} 2-\mathrm{Si} 21$ & $110.47(6)$ \\
\hline $\mathrm{Si} 23-\mathrm{Si} 2-\mathrm{Si} 21$ & $108.66(3)$ \\
\hline $\mathrm{O} 2-\mathrm{Si} 2-\mathrm{Si} 22$ & $111.86(6)$ \\
\hline $\mathrm{Si} 23-\mathrm{Si} 2-\mathrm{Si} 22$ & $108.39(3)$ \\
\hline $\mathrm{Si} 21-\mathrm{Si} 2-\mathrm{Si} 22$ & $105.39(3)$ \\
\hline $\mathrm{O} 3-\mathrm{Si} 3-\mathrm{Si} 31$ & $108.71(6)$ \\
\hline $\mathrm{O} 3-\mathrm{Si} 3-\mathrm{Si} 32$ & $111.10(6)$ \\
\hline $\mathrm{Si} 31-\mathrm{Si} 3-\mathrm{Si} 32$ & $107.87(3)$ \\
\hline $\mathrm{O} 3-\mathrm{Si} 3-\mathrm{Si} 33$ & $114.87(6)$ \\
\hline $\mathrm{Si} 31-\mathrm{Si} 3-\mathrm{Si} 33$ & $104.51(3)$ \\
\hline $\mathrm{Si} 32-\mathrm{Si} 3$-Si33 & $109.37(3)$ \\
\hline C111-Si11-C113 & $107.83(14)$ \\
\hline $\mathrm{C} 111-\mathrm{Si} 11-\mathrm{C} 112$ & $106.33(14)$ \\
\hline C113-Si11-C112 & $107.66(13)$ \\
\hline C111—Si11-Si1 & $116.00(10)$ \\
\hline C113-Si11-Si1 & $104.97(10)$ \\
\hline C112-Si11-Si1 & $113.65(9)$ \\
\hline $\mathrm{C} 122-\mathrm{Si} 12-\mathrm{C} 121$ & $106.80(16)$ \\
\hline $\mathrm{C} 122-\mathrm{Si} 12-\mathrm{C} 123$ & $112.6(2)$ \\
\hline $\mathrm{C} 121-\mathrm{Si} 12-\mathrm{C} 123$ & $105.71(16)$ \\
\hline C122-Si12-Si1 & $111.92(11)$ \\
\hline C121-Si12-Si1 & $115.15(10)$ \\
\hline C123-Si12-Si1 & $104.60(12)$ \\
\hline $\mathrm{C} 132-\mathrm{Si13}-\mathrm{C} 131$ & $108.65(14)$ \\
\hline $\mathrm{C} 132-\mathrm{Si13}-\mathrm{C} 133$ & $108.52(16)$ \\
\hline $\mathrm{C} 131-\mathrm{Si13}-\mathrm{C} 133$ & $108.79(14)$ \\
\hline C132-Si13-Si1 & $109.36(9)$ \\
\hline C131-Si13-Si1 & $107.27(10)$ \\
\hline C133-Si13-Si1 & $114.13(11)$ \\
\hline $\mathrm{C} 212-\mathrm{Si} 21-\mathrm{C} 211$ & $108.89(14)$ \\
\hline $\mathrm{C} 212-\mathrm{Si} 21-\mathrm{C} 213$ & $108.26(16)$ \\
\hline
\end{tabular}

\begin{tabular}{|c|c|}
\hline $\mathrm{Si13}-\mathrm{C} 133-\mathrm{H} 13 \mathrm{I}$ & 109.5 \\
\hline $\mathrm{H} 13 \mathrm{G}-\mathrm{C} 133-\mathrm{H} 13 \mathrm{I}$ & 109.5 \\
\hline $\mathrm{H} 13 \mathrm{H}-\mathrm{C} 133-\mathrm{H} 13 \mathrm{I}$ & 109.5 \\
\hline $\mathrm{Si} 21-\mathrm{C} 211-\mathrm{H} 21 \mathrm{~A}$ & 109.5 \\
\hline $\mathrm{Si} 21-\mathrm{C} 211-\mathrm{H} 21 \mathrm{~B}$ & 109.5 \\
\hline $\mathrm{H} 21 \mathrm{~A}-\mathrm{C} 211-\mathrm{H} 21 \mathrm{~B}$ & 109.5 \\
\hline $\mathrm{Si} 21-\mathrm{C} 211-\mathrm{H} 21 \mathrm{C}$ & 109.5 \\
\hline $\mathrm{H} 21 \mathrm{~A}-\mathrm{C} 211-\mathrm{H} 21 \mathrm{C}$ & 109.5 \\
\hline $\mathrm{H} 21 \mathrm{~B}-\mathrm{C} 211-\mathrm{H} 21 \mathrm{C}$ & 109.5 \\
\hline $\mathrm{Si} 21-\mathrm{C} 212-\mathrm{H} 21 \mathrm{D}$ & 109.5 \\
\hline $\mathrm{Si} 21-\mathrm{C} 212-\mathrm{H} 21 \mathrm{E}$ & 109.5 \\
\hline $\mathrm{H} 21 \mathrm{D}-\mathrm{C} 212-\mathrm{H} 21 \mathrm{E}$ & 109.5 \\
\hline $\mathrm{Si} 21-\mathrm{C} 212-\mathrm{H} 21 \mathrm{~F}$ & 109.5 \\
\hline $\mathrm{H} 21 \mathrm{D}-\mathrm{C} 212-\mathrm{H} 21 \mathrm{~F}$ & 109.5 \\
\hline $\mathrm{H} 21 \mathrm{E}-\mathrm{C} 212-\mathrm{H} 21 \mathrm{~F}$ & 109.5 \\
\hline $\mathrm{Si} 21-\mathrm{C} 213-\mathrm{H} 21 \mathrm{G}$ & 109.5 \\
\hline $\mathrm{Si} 21-\mathrm{C} 213-\mathrm{H} 21 \mathrm{H}$ & 109.5 \\
\hline $\mathrm{H} 21 \mathrm{G}-\mathrm{C} 213-\mathrm{H} 21 \mathrm{H}$ & 109.5 \\
\hline $\mathrm{Si} 21-\mathrm{C} 213-\mathrm{H} 21 \mathrm{I}$ & 109.5 \\
\hline $\mathrm{H} 21 \mathrm{G}-\mathrm{C} 213-\mathrm{H} 21 \mathrm{I}$ & 109.5 \\
\hline $\mathrm{H} 21 \mathrm{H}-\mathrm{C} 213-\mathrm{H} 21 \mathrm{I}$ & 109.5 \\
\hline $\mathrm{Si} 22-\mathrm{C} 221-\mathrm{H} 22 \mathrm{~A}$ & 109.5 \\
\hline $\mathrm{Si} 22-\mathrm{C} 221-\mathrm{H} 22 \mathrm{~B}$ & 109.5 \\
\hline $\mathrm{H} 22 \mathrm{~A}-\mathrm{C} 221-\mathrm{H} 22 \mathrm{~B}$ & 109.5 \\
\hline $\mathrm{Si} 22-\mathrm{C} 221-\mathrm{H} 22 \mathrm{C}$ & 109.5 \\
\hline $\mathrm{H} 22 \mathrm{~A}-\mathrm{C} 221-\mathrm{H} 22 \mathrm{C}$ & 109.5 \\
\hline $\mathrm{H} 22 \mathrm{~B}-\mathrm{C} 221-\mathrm{H} 22 \mathrm{C}$ & 109.5 \\
\hline $\mathrm{Si} 22-\mathrm{C} 222-\mathrm{H} 22 \mathrm{D}$ & 109.5 \\
\hline $\mathrm{Si} 22-\mathrm{C} 222-\mathrm{H} 22 \mathrm{E}$ & 109.5 \\
\hline $\mathrm{H} 22 \mathrm{D}-\mathrm{C} 222-\mathrm{H} 22 \mathrm{E}$ & 109.5 \\
\hline $\mathrm{Si} 22-\mathrm{C} 222-\mathrm{H} 22 \mathrm{~F}$ & 109.5 \\
\hline $\mathrm{H} 22 \mathrm{D}-\mathrm{C} 222-\mathrm{H} 22 \mathrm{~F}$ & 109.5 \\
\hline $\mathrm{H} 22 \mathrm{E}-\mathrm{C} 222-\mathrm{H} 22 \mathrm{~F}$ & 109.5 \\
\hline $\mathrm{Si} 22-\mathrm{C} 223-\mathrm{H} 22 \mathrm{G}$ & 109.5 \\
\hline $\mathrm{Si} 22-\mathrm{C} 223-\mathrm{H} 22 \mathrm{H}$ & 109.5 \\
\hline $\mathrm{H} 22 \mathrm{G}-\mathrm{C} 223-\mathrm{H} 22 \mathrm{H}$ & 109.5 \\
\hline $\mathrm{Si} 22-\mathrm{C} 223-\mathrm{H} 22 \mathrm{I}$ & 109.5 \\
\hline $\mathrm{H} 22 \mathrm{G}-\mathrm{C} 223-\mathrm{H} 22 \mathrm{I}$ & 109.5 \\
\hline $\mathrm{H} 22 \mathrm{H}-\mathrm{C} 223-\mathrm{H} 22 \mathrm{I}$ & 109.5 \\
\hline $\mathrm{Si} 23-\mathrm{C} 231-\mathrm{K} 1^{\mathrm{i}}$ & $138.83(13)$ \\
\hline $\mathrm{Si} 23-\mathrm{C} 231-\mathrm{H} 23 \mathrm{~A}$ & 109.5 \\
\hline $\mathrm{K} 1 \mathrm{i}-\mathrm{C} 231-\mathrm{H} 23 \mathrm{~A}$ & 111.6 \\
\hline $\mathrm{Si} 23-\mathrm{C} 231-\mathrm{H} 23 \mathrm{~B}$ & 109.5 \\
\hline 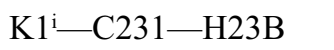 & 53.8 \\
\hline $\mathrm{H} 23 \mathrm{~A}-\mathrm{C} 231-\mathrm{H} 23 \mathrm{~B}$ & 109.5 \\
\hline $\mathrm{Si} 23-\mathrm{C} 231-\mathrm{H} 23 \mathrm{C}$ & 109.5 \\
\hline $\mathrm{K} 11^{\mathrm{i}}-\mathrm{C} 231-\mathrm{H} 23 \mathrm{C}$ & 58.0 \\
\hline $\mathrm{H} 23 \mathrm{~A}-\mathrm{C} 231-\mathrm{H} 23 \mathrm{C}$ & 109.5 \\
\hline
\end{tabular}




\begin{tabular}{|c|c|}
\hline $\mathrm{C} 211-\mathrm{Si} 21-\mathrm{C} 213$ & $106.10(13)$ \\
\hline $\mathrm{C} 212-\mathrm{Si} 21-\mathrm{Si} 2$ & $106.61(11)$ \\
\hline $\mathrm{C} 211-\mathrm{Si} 21-\mathrm{Si} 2$ & $111.23(9)$ \\
\hline $\mathrm{C} 213-\mathrm{Si} 21-\mathrm{Si} 2$ & $115.60(10)$ \\
\hline $\mathrm{C} 221-\mathrm{Si} 22-\mathrm{C} 223$ & $109.79(17)$ \\
\hline $\mathrm{C} 221-\mathrm{Si} 22-\mathrm{C} 222$ & $108.03(17)$ \\
\hline $\mathrm{C} 223-\mathrm{Si} 22-\mathrm{C} 222$ & $104.79(17)$ \\
\hline $\mathrm{C} 221-\mathrm{Si} 22-\mathrm{Si} 2$ & $108.00(11)$ \\
\hline $\mathrm{C} 223-\mathrm{Si} 22-\mathrm{Si} 2$ & $113.84(12)$ \\
\hline $\mathrm{C} 222-\mathrm{Si} 22-\mathrm{Si} 2$ & $112.23(11)$ \\
\hline $\mathrm{C} 232-\mathrm{Si} 23-\mathrm{C} 231$ & $109.51(13)$ \\
\hline $\mathrm{C} 232-\mathrm{Si} 23-\mathrm{C} 233$ & $106.12(15)$ \\
\hline $\mathrm{C} 231-\mathrm{Si} 23-\mathrm{C} 233$ & $109.29(15)$ \\
\hline $\mathrm{C} 232-\mathrm{Si} 23-\mathrm{Si} 2$ & $107.82(11)$ \\
\hline $\mathrm{C} 231-\mathrm{Si} 23-\mathrm{Si} 2$ & $110.18(9)$ \\
\hline C233-Si23-Si2 & $113.77(11)$ \\
\hline C312-Si31-C311 & $106.59(17)$ \\
\hline $\mathrm{C} 312-\mathrm{Si} 31-\mathrm{C} 313$ & $109.72(14)$ \\
\hline $\mathrm{C} 311-\mathrm{Si} 31-\mathrm{C} 313$ & $107.41(14)$ \\
\hline $\mathrm{C} 312-\mathrm{Si3} 1-\mathrm{Si} 3$ & $112.33(11)$ \\
\hline $\mathrm{C} 311-\mathrm{Si} 31-\mathrm{Si} 3$ & $114.40(11)$ \\
\hline $\mathrm{C} 313-\mathrm{Si} 31-\mathrm{Si} 3$ & $106.26(9)$ \\
\hline $\mathrm{C} 323-\mathrm{Si} 32-\mathrm{C} 322$ & $106.93(15)$ \\
\hline $\mathrm{C} 323-\mathrm{Si} 32-\mathrm{C} 321$ & $107.74(15)$ \\
\hline C322-Si32-C321 & $107.65(16)$ \\
\hline $\mathrm{C} 323-\mathrm{Si} 32-\mathrm{Si} 3$ & $110.59(9)$ \\
\hline $\mathrm{C} 322-\mathrm{Si} 32-\mathrm{Si} 3$ & $114.41(10)$ \\
\hline $\mathrm{C} 321-\mathrm{Si32}-\mathrm{Si} 3$ & $109.27(10)$ \\
\hline C333-Si33-C331 & $107.17(17)$ \\
\hline C333-Si33-C332 & $107.22(14)$ \\
\hline $\mathrm{C} 331-\mathrm{Si} 33-\mathrm{C} 332$ & $107.23(14)$ \\
\hline C333-Si33-Si3 & $114.17(10)$ \\
\hline C331-Si33-Si3 & $112.84(11)$ \\
\hline $\mathrm{C} 332-\mathrm{Si} 33-\mathrm{Si} 3$ & $107.87(9)$ \\
\hline $\mathrm{O} 3-\mathrm{A} 11-\mathrm{O} 2$ & $114.11(8)$ \\
\hline $\mathrm{O} 3-\mathrm{A} 11-\mathrm{O} 1$ & $114.09(8)$ \\
\hline $\mathrm{O} 2-\mathrm{A} 11-\mathrm{O} 1$ & $114.64(7)$ \\
\hline $\mathrm{O} 3-\mathrm{Al} 1-\mathrm{Cl} 1$ & $104.39(6)$ \\
\hline $\mathrm{O} 2-\mathrm{A} 11-\mathrm{Cl} 1$ & $107.48(6)$ \\
\hline $\mathrm{O} 1-\mathrm{A} 11-\mathrm{Cl} 1$ & $100.32(6)$ \\
\hline $\mathrm{O} 3-\mathrm{A} 11-\mathrm{K} 1$ & $114.34(6)$ \\
\hline $\mathrm{O} 2-\mathrm{A} 11-\mathrm{K} 1$ & $131.25(6)$ \\
\hline $\mathrm{O} 1-\mathrm{A} 11-\mathrm{K} 1$ & $46.33(5)$ \\
\hline $\mathrm{C} 11-\mathrm{A} 11-\mathrm{K} 1$ & $54.69(2)$ \\
\hline Si1-O1-Al1 & $146.69(10)$ \\
\hline Sil-O1-K1 & $104.85(7)$ \\
\hline $\mathrm{A} 11-\mathrm{O} 1-\mathrm{K} 1$ & $106.56(6)$ \\
\hline $\mathrm{Si} 2-\mathrm{O} 2-\mathrm{A} 11$ & $156.49(10)$ \\
\hline
\end{tabular}

$\mathrm{H} 23 \mathrm{~B}-\mathrm{C} 231-\mathrm{H} 23 \mathrm{C} \quad 109.5$

$\mathrm{Si} 23-\mathrm{C} 232-\mathrm{H} 23 \mathrm{D} \quad 109.5$

$\mathrm{Si} 23-\mathrm{C} 232-\mathrm{H} 23 \mathrm{E} \quad 109.5$

$\mathrm{H} 23 \mathrm{D}-\mathrm{C} 232-\mathrm{H} 23 \mathrm{E} \quad 109.5$

$\mathrm{Si} 23-\mathrm{C} 232-\mathrm{H} 23 \mathrm{~F} \quad 109.5$

$\mathrm{H} 23 \mathrm{D}-\mathrm{C} 232-\mathrm{H} 23 \mathrm{~F} \quad 109.5$

$\mathrm{H} 23 \mathrm{E}-\mathrm{C} 232-\mathrm{H} 23 \mathrm{~F} \quad 109.5$

$\mathrm{Si} 23-\mathrm{C} 233-\mathrm{H} 23 \mathrm{G} \quad 109.5$

$\mathrm{Si} 23-\mathrm{C} 233-\mathrm{H} 23 \mathrm{H} \quad 109.5$

$\mathrm{H} 23 \mathrm{G}-\mathrm{C} 233-\mathrm{H} 23 \mathrm{H} \quad 109.5$

$\mathrm{Si} 23-\mathrm{C} 233-\mathrm{H} 23 \mathrm{I} \quad 109.5$

$\mathrm{H} 23 \mathrm{G}-\mathrm{C} 233-\mathrm{H} 23 \mathrm{I} \quad 109.5$

$\mathrm{H} 23 \mathrm{H}-\mathrm{C} 233-\mathrm{H} 23 \mathrm{I}$

$\mathrm{Si} 31-\mathrm{C} 311-\mathrm{H} 31 \mathrm{~A} \quad 109.5$

$\mathrm{Si} 31-\mathrm{C} 311-\mathrm{H} 31 \mathrm{~B} \quad 109.5$

H31A-C311-H31B $\quad 109.5$

$\mathrm{Si31}-\mathrm{C} 311-\mathrm{H} 31 \mathrm{C} \quad 109.5$

$\mathrm{H} 31 \mathrm{~A}-\mathrm{C} 311-\mathrm{H} 31 \mathrm{C} \quad 109.5$

$\mathrm{H} 31 \mathrm{~B}-\mathrm{C} 311-\mathrm{H} 31 \mathrm{C} \quad 109.5$

Si31-C312-H31D $\quad 109.5$

$\mathrm{Si} 31-\mathrm{C} 312-\mathrm{H} 31 \mathrm{E} \quad 109.5$

$\mathrm{H} 31 \mathrm{D}-\mathrm{C} 312-\mathrm{H} 31 \mathrm{E} \quad 109.5$

$\mathrm{Si31}-\mathrm{C} 312-\mathrm{H} 31 \mathrm{~F} \quad 109.5$

$\mathrm{H} 31 \mathrm{D}-\mathrm{C} 312-\mathrm{H} 31 \mathrm{~F} \quad 109.5$

$\mathrm{H} 31 \mathrm{E}-\mathrm{C} 312-\mathrm{H} 31 \mathrm{~F} \quad 109.5$

$\mathrm{Si31}-\mathrm{C} 313-\mathrm{K} 1 \quad 145.40$ (12)

$\mathrm{Si} 31-\mathrm{C} 313-\mathrm{H} 31 \mathrm{G} \quad 109.5$

$\mathrm{K} 1-\mathrm{C} 313-\mathrm{H} 31 \mathrm{G} \quad 104.6$

$\mathrm{Si} 31-\mathrm{C} 313-\mathrm{H} 31 \mathrm{H} \quad 109.5$

$\mathrm{K} 1-\mathrm{C} 313-\mathrm{H} 31 \mathrm{H} \quad 63.3$

$\mathrm{H} 31 \mathrm{G}-\mathrm{C} 313-\mathrm{H} 31 \mathrm{H} \quad 109.5$

$\mathrm{Si} 31-\mathrm{C} 313-\mathrm{H} 31 \mathrm{I} \quad 109.5$

$\mathrm{K} 1-\mathrm{C} 313-\mathrm{H} 31 \mathrm{I} \quad 51.3$

$\mathrm{H} 31 \mathrm{G}-\mathrm{C} 313-\mathrm{H} 31 \mathrm{I} \quad 109.5$

$\mathrm{H} 31 \mathrm{H}-\mathrm{C} 313-\mathrm{H} 31 \mathrm{I}$

$\mathrm{Si} 32-\mathrm{C} 321-\mathrm{H} 32 \mathrm{~A} \quad 109.5$

$\mathrm{Si32}-\mathrm{C} 321-\mathrm{H} 32 \mathrm{~B} \quad 109.5$

$\mathrm{H} 32 \mathrm{~A}-\mathrm{C} 321-\mathrm{H} 32 \mathrm{~B} \quad 109.5$

$\mathrm{Si} 32-\mathrm{C} 321-\mathrm{H} 32 \mathrm{C} \quad 109.5$

$\mathrm{H} 32 \mathrm{~A}-\mathrm{C} 321-\mathrm{H} 32 \mathrm{C} \quad 109.5$

$\mathrm{H} 32 \mathrm{~B}-\mathrm{C} 321-\mathrm{H} 32 \mathrm{C} \quad 109.5$

$\mathrm{Si32}-\mathrm{C} 322-\mathrm{H} 32 \mathrm{D} \quad 109.5$

$\mathrm{Si32}-\mathrm{C} 322-\mathrm{H} 32 \mathrm{E} \quad 109.5$

$\mathrm{H} 32 \mathrm{D}-\mathrm{C} 322-\mathrm{H} 32 \mathrm{E} \quad 109.5$

$\mathrm{Si} 32-\mathrm{C} 322-\mathrm{H} 32 \mathrm{~F} \quad 109.5$

$\mathrm{H} 32 \mathrm{D}-\mathrm{C} 322-\mathrm{H} 32 \mathrm{~F} \quad 109.5$

$\mathrm{H} 32 \mathrm{E}-\mathrm{C} 322-\mathrm{H} 32 \mathrm{~F} \quad 109.5$

$\mathrm{Si} 32-\mathrm{C} 323-\mathrm{H} 32 \mathrm{G} \quad 109.5$ 
$\mathrm{Si3}-\mathrm{O} 3-\mathrm{Al1}$

Si11-C111-H11A

Si11-C111-H11B

H11A-C111-H11B

Si11-C111-H11C

$\mathrm{H} 11 \mathrm{~A}-\mathrm{C} 111-\mathrm{H} 11 \mathrm{C}$

H11B - C111-H11C

Si11-C112-K1

Si11-C112-H11D

K1-C112-H11D

Si11-C112-H11E

$\mathrm{K} 1-\mathrm{C} 112-\mathrm{H} 11 \mathrm{E}$

H11D-C112-H11E

Si11-C112-H11F

$\mathrm{K} 1-\mathrm{C} 112-\mathrm{H} 11 \mathrm{~F}$

H11D-C112-H11F

H11E-C112-H11F

Si11-C113-H11G

Si11-C113-H11H

$\mathrm{H} 11 \mathrm{G}-\mathrm{C} 113-\mathrm{H} 11 \mathrm{H}$

Si11-C113-H11I

$\mathrm{H} 11 \mathrm{G}-\mathrm{C} 113-\mathrm{H} 11 \mathrm{I}$

$\mathrm{H} 11 \mathrm{H}-\mathrm{C} 113-\mathrm{H} 11 \mathrm{I}$

Si13-Si1-O1-Al1

Si11-Si1-O1-Al1

Si12-Si1-O1-A11

K1-Si1-O1-Al1

Si13-Si1-O1-K1

Si11-Si1-O1-K1

Si12-Si1-O1-K1

O3-Al1-O1-Si1

O2-A11-O1-Si1

Cl1-Al1-O1-Si1

K1-Al1-O1-Si1

O3-Al1-O1-K1

$\mathrm{O} 2-\mathrm{A} 11-\mathrm{O} 1-\mathrm{K} 1$

Cl1-Al1-O1-K1

$\mathrm{Si} 23-\mathrm{Si} 2-\mathrm{O} 2-\mathrm{A} 11$

$\mathrm{Si} 21-\mathrm{Si} 2-\mathrm{O} 2-\mathrm{A} 11$

$\mathrm{Si} 22-\mathrm{Si} 2-\mathrm{O} 2-\mathrm{A} 11$

$\mathrm{O} 3-\mathrm{A} 11-\mathrm{O} 2-\mathrm{Si} 2$

$\mathrm{O} 1-\mathrm{Al1}-\mathrm{O} 2-\mathrm{Si} 2$
$156.75(10)$

109.5

109.5

109.5

109.5

109.5

109.5

$98.58(10)$

109.5

151.9

109.5

56.9

109.5

109.5

61.0

109.5

109.5

109.5

109.5

109.5

109.5

109.5

109.5

$-22.36(19)$

$-145.50(15)$

97.37 (17)

$160.1(2)$

177.54 (4)

$54.39(7)$

$-62.73(6)$

98.69 (18)

$-35.5(2)$

$-150.32(16)$

$-159.9(2)$

$-101.38(8)$

$124.41(7)$

$9.61(6)$

$118.7(2)$

$-2.4(3)$

$-119.5(2)$

63.4 (3)

$-162.4(2)$
$\mathrm{Si} 32-\mathrm{C} 323-\mathrm{H} 32 \mathrm{H}$

$\mathrm{H} 32 \mathrm{G}-\mathrm{C} 323-\mathrm{H} 32 \mathrm{H}$

$\mathrm{Si32}-\mathrm{C} 323-\mathrm{H} 32 \mathrm{I}$

$\mathrm{H} 32 \mathrm{G}-\mathrm{C} 323-\mathrm{H} 32 \mathrm{I}$

$\mathrm{H} 32 \mathrm{H}-\mathrm{C} 323-\mathrm{H} 32 \mathrm{I}$

Si33-C331-H33A

Si33-C $331-\mathrm{H} 33 \mathrm{~B}$

H33A-C $331-\mathrm{H} 33 \mathrm{~B}$

Si33-C $331-\mathrm{H} 33 \mathrm{C}$

$\mathrm{H} 33 \mathrm{~A}-\mathrm{C} 331-\mathrm{H} 33 \mathrm{C}$

$\mathrm{H} 33 \mathrm{~B}-\mathrm{C} 331-\mathrm{H} 33 \mathrm{C}$

Si33-C332-H33D

Si33-C332-H33E

H33D-C $332-\mathrm{H} 33 \mathrm{E}$

Si33-C332-H33F

$\mathrm{H} 33 \mathrm{D}-\mathrm{C} 332-\mathrm{H} 33 \mathrm{~F}$

H33E-C332-H33F

Si33-C333-H33G

$\mathrm{Si} 33-\mathrm{C} 333-\mathrm{H} 33 \mathrm{H}$

$\mathrm{H} 33 \mathrm{G}-\mathrm{C} 333-\mathrm{H} 33 \mathrm{H}$

Si33-C333-H33I

H33G-C $333-\mathrm{H} 33 \mathrm{I}$

$\mathrm{H} 33 \mathrm{H}-\mathrm{C} 333-\mathrm{H} 33 \mathrm{I}$

$\mathrm{C} 11-\mathrm{A} 11-\mathrm{O} 2-\mathrm{Si} 2$

$\mathrm{K} 1-\mathrm{A} 11-\mathrm{O} 2-\mathrm{Si} 2$

$\mathrm{Si} 31-\mathrm{Si3}-\mathrm{O} 3-\mathrm{A} 11$

$\mathrm{Si} 32-\mathrm{Si} 3-\mathrm{O} 3-\mathrm{Al}$

$\mathrm{Si33}-\mathrm{Si} 3-\mathrm{O} 3-\mathrm{Al}$

$\mathrm{O} 2-\mathrm{A} 11-\mathrm{O} 3-\mathrm{Si} 3$

$\mathrm{O} 1-\mathrm{A} 11-\mathrm{O} 3-\mathrm{Si} 3$

$\mathrm{C} 11-\mathrm{A} 11-\mathrm{O} 3-\mathrm{Si} 3$

$\mathrm{K} 1-\mathrm{A} 11-\mathrm{O} 3-\mathrm{Si} 3$

C111-Si11-C112-K1

C113-Si11-C112-K1

Si1-Si11-C112-K1

$\mathrm{C} 232-\mathrm{Si} 23-\mathrm{C} 231-\mathrm{K} 1^{\mathrm{i}}$

$\mathrm{C} 233-\mathrm{Si} 23-\mathrm{C} 231-\mathrm{K} 1^{\mathrm{i}}$

$\mathrm{Si} 2-\mathrm{Si} 23-\mathrm{C} 231-\mathrm{K} 1^{\mathrm{i}}$

C312-Si31-C313-K1

C311-Si31-C313-K1

Si3-Si31-C313-K1
109.5

109.5

109.5

109.5

109.5

109.5

109.5

109.5

109.5

109.5

109.5

109.5

109.5

109.5

109.5

109.5

109.5

109.5

109.5

109.5

109.5

109.5

109.5

$-51.9(3)$

$-109.9(2)$

$-62.1(3)$

179.4 (2)

$54.6(3)$

$-151.8(3)$

73.8 (3)

-34.7 (3)

22.7 (3)

$-114.26(11)$

$130.39(12)$

14.58 (11)

$115.2(2)$

$-129.0(2)$

$-3.3(2)$

110.5 (2)

-134.0 (2)

-11.2 (3)

Symmetry code: (i) $-x+1,-y+1,-z+1$. 